\title{
AN INTRODUCTION TO SPHERICAL ORBIT SPACES
}

\author{
JILL MCGOWAN and CATHERINE SEARLE
}

Received 21 February 2001 and in revised form 24 September 2001

Consider a compact, connected Lie group $G$ acting isometrically on a sphere $S^{n}$ of radius 1 . Two-dimensional quotient spaces of the type $S^{n} / G$ have been investigated extensively. This paper provides an elementary introduction, for nonspecialists, to this important field by way of several classical examples and supplies an explicit list of all the isotropy subgroups involved in these examples.

2000 Mathematics Subject Classification: 53C20, 57S15.

1. Introduction. Spheres have long been an active area of investigation for geometers, algebraists, and physicists because of the richness of symmetry and beguiling simplicity they offer. In particular, a sphere is associated with an enormous group of transformations that preserve its underlying metric; these transformations are called isometries. Careful study of these actions has led to deeper understanding of spherical geometry, to the discovery of many examples of minimal submanifolds, and hypersurfaces on Stiefel manifolds and Grassmannian manifolds. Moreover, it is interesting to see how simple linear algebra reveals the secrets of the geometry of these orbit spaces.

Before describing specifically the content of this paper, we present a motivating example.

EXAMPLE 1.1. Suppose that we allow matrices of the form

$$
g=\left[\begin{array}{ccc}
\cos \theta & -\sin \theta & 0 \\
\sin \theta & \cos \theta & 0 \\
0 & 0 & 1
\end{array}\right]
$$

to operate on $S^{2}=\left\{(x, y, z): x^{2}+y^{2}+z^{2}=1\right\}$ by matrix multiplication. This is a rotation around the $z$-axis by an angle $\theta$. There is an obvious isomorphism between this group $G$ of matrices $g$ and the circle $S^{1}=\left\{e^{i \theta}: \theta \in[0,2 \pi)\right\}$, so we will think of this action as an action of $S^{1}$ on $S^{2}$. Given a point $(x, y, z) \in S^{2}$, we consider the collection of all the images of this point under multiplication by elements $g$ of $S^{1}$. That is, consider $G((x, y, z))=\{g((x, y, z)): g \in G\}$. (This set is called the orbit of $(x, y, z)$.) As no matrix of this type changes the value of $z$, the orbit is the intersection of a plane $z=a$ with the two-sphere: a circle centered on the $z$-axis and contained in $S^{2}$. These sets, the orbits of the points in $S^{2}$, decompose $S^{2}$ into disjoint sets, they therefore define an equivalence relation on $S^{2}$. We consider these sets as the points in a new space $S^{2} / S^{1}$, called the orbit space of the $S^{1}$ action on $S^{2}$. This action fixes 


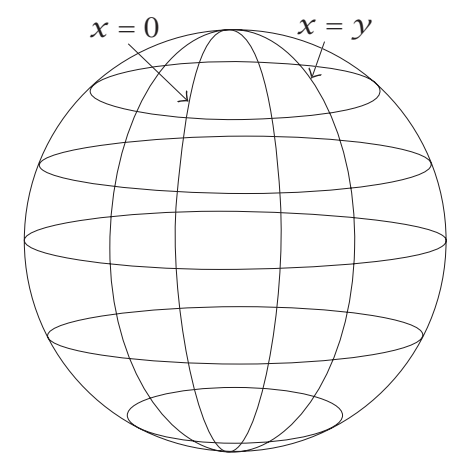

FIGURE 1.1

the $x y$-plane in $\mathbb{R}^{3}$, since

$$
\left[\begin{array}{ccc}
\cos \theta & -\sin \theta & 0 \\
\sin \theta & \cos \theta & 0 \\
0 & 0 & 1
\end{array}\right]\left[\begin{array}{l}
x \\
y \\
0
\end{array}\right]=\left[\begin{array}{c}
x \cos \theta-y \sin \theta \\
x \sin \theta+y \cos \theta \\
0
\end{array}\right] .
$$

It also fixes the normal space, the $z$-axis. Because the action fixes these two normal subspaces, it is called reducible. If we intersect the sphere with the half plane $\{(x, 0, z)$ : $x \geq 0$, we have exactly one point in the sphere for each orbit. We see then that the orbit space is an interval, isometric to a closed half-circle of radius one.

Distance in the orbit space is defined to be the distance between orbits in the original space. Since the distance between the north pole and the south pole in $S^{2}$ is $\pi$ (half the circumference of a circle in $S^{2}$ joining them), this is the length of the orbit space. It is not a manifold (although it is a manifold with boundary) because of the endpoints. (See Figure 1.1.)

In this paper, we see how to generalize the techniques used in analyzing the example above to actions on $n$-dimensional spheres. We will work out several cases, as described below. In brief, the contents of this paper are as follows. In Section 2, we begin with some necessary definitions. In Section 3, we offer some background and examples from classical theory on group actions on spheres. In Section 4, we describe in detail the following reducible actions on spheres: $(G, \phi)=\left(\operatorname{SO}\left(n_{1}\right) \times\right.$ $\left.\mathrm{SO}\left(n_{2}\right) \times \mathrm{SO}\left(n_{3}\right), \rho_{n_{1}}+\rho_{n_{2}}+\rho_{n_{3}}\right)$, where $\rho_{n}$ is the standard representation of $\mathrm{SO}(n)$, $(G, \phi)=\left(\mathrm{SO}(2) \times \mathrm{SO}(m), \rho_{2} \otimes \rho_{m}+1\right)$, where 1 is a one-dimensional trivial representation, and $(G, \phi)=\left(\operatorname{SO}(k), 2 \rho_{k}\right)$, for $k \geq 3$. (Even though the groups in these three cases are getting smaller, the actions are actually becoming more complicated, as we shall see. The subset of GL $(n, \mathbb{R})$ for which all elements $A$ satisfy $A^{-1}=A^{T}$ is called $\mathrm{O}(n)$; is its connected component of the identity.) In Section 5, we describe the following irreducible actions: $(G, \phi)=\left(\mathrm{SO}(3) \times \mathrm{SO}(n), \rho_{3} \otimes \rho_{n}\right),(G, \phi)=\left(\mathrm{SO}(3) \times \mathrm{SO}(3), \rho_{3} \otimes \rho_{3}\right)$, $(G, \phi)=\left(\mathrm{U}(3) \times \mathrm{SU}(n), \rho_{2} \times \mu_{3} \otimes \mu_{n}\right)$, and $(G, \phi)=\left(\mathrm{Sp}(3) \times \mathrm{Sp}(n), v_{3} \otimes v_{n}\right)$. In Section 6 , we offer our conclusions and a suggestion for new areas of investigation. This method for finding these isotropy subgroups is of interest because of the understanding it lends to classical Lie groups and certain manifolds associated with them, which are 
of concern to physicists as well as geometers. We hope this method makes this information more accessible to physicists and nonspecialists who may have an interest in these orbit spaces. Moreover, the decompositions themselves are of interest, in that many of them are not well known, especially those that correspond to irreducible actions.

Hsiang and Lawson's classical paper on this subject [4] describes most of the orbit spaces produced by irreducible, maximal linear groups on the standard sphere and computes the principal isotropy subgroups in each case. Straume's comprehensive work on the subject $[9,10]$ describes not only all these actions in detail, but also actions on homotopy spheres as well. In these, he completes the proof of the classification of spheres with orbit spaces of dimensions 1 and 2. He provides the list of orbit spaces of those cases omitted by Hsiang and Lawson in [8]. Both [4, 9] list the principal isotropy groups involved and, in the case of the polar actions, the associated symmetric spaces as well. The intermediate isotropy groups (i.e., those on the edges and vertices of the orbit spaces) are not explicitly included in any of these papers; however, they may be computed using the configuration of the spherical triangle in question and the list of possible cohomogeneity-one actions.

With respect to the specific examples included herein, except for the $\mathrm{SO}(3) \times \mathrm{SO}(3)$ example, Hsiang and Lawson [4] list all the orbit spaces resulting from the irreducible actions in Section 5 applied to $\mathbb{R}^{n}$, and their principal isotropy subgroups. In addition, they describe the orbit space resulting from the reducible $\mathrm{SO}(k)$ action on $\mathbb{R}^{n}$, where the isotropy subgroups are also listed. Straume [8] also lists the orbit space resulting from this $\mathrm{SO}(k)$ action on $S^{2 n-1}$. While the orbit space resulting from the $\mathrm{SO}(3) \times \mathrm{SO}(3)$ action on $S^{8}$ can be deduced by reference to all three of $[8,9,10]$, it is nowhere explicitly calculated. (Hsiang and Lawson [4] do include an explicit statement about the orbit space $S^{8} / \mathrm{SO}(3) \times \mathrm{SO}(3)$, but they erroneously list it as the same orbit space as $S^{3 n-1} / \mathrm{SO}(3) \times \mathrm{SO}(n)$ for $n>3$.) The orbit spaces of the first two reducible actions in Section 4 are not listed anywhere, as they are very easy to compute from the cohomogeneity-one actions, which $[4,9,10]$ classify.

2. Definitions. In our introduction, we gave some rough definitions. Here, we will formalize some of them and add a few others. All the actions we will consider are actions on spheres which, of course, are manifolds.

DEFINITION 2.1. An $n$-dimensional manifold $M$ is Hausdorff topological space furnished with a collection of open sets $U_{\alpha}$, such that

(1) $M=\bigcup_{\alpha \in A} U_{\alpha}$;

(2) on any open set $U_{\alpha}$, there is a homeomorphism, $\phi_{\alpha}$, from $U_{\alpha}$ to $\mathbb{R}^{n}$, such that, whenever the intersection of any pair of these open sets $U_{\alpha} \cap U_{\beta}$ is nonempty, $\left.\phi_{\alpha} \circ \phi_{\beta}{ }^{-1}\right|_{\phi_{\beta}\left(U_{\alpha} \cap U_{\beta}\right)}$ is a smooth $\left(C^{\infty}\right)$ homeomorphism between subsets of $\mathbb{R}^{n}$.

Some manifolds have other special properties as well. On any $n$-dimensional Riemannian manifold $M$, there are several naturally associated functions, such as curvature and distance [5]. For this reason, certain numbers come to be associated with a manifold. For instance, on a manifold whose curvature is constant, like a sphere, that constant is an invariant (under isometry) of the manifold. 
Since we restrict our attention to the standard sphere $S^{n}$ embedded in $\mathbb{R}^{n+1}$, for the sake of simplicity in the next definition, we assume our manifold to be embedded in Euclidean space, where we have a well-defined notion of arclength.

Definition 2.2. The distance $d(x, y)$ between two points $x$ and $y$ in $M$ is the infimum of the lengths of all piecewise smooth curves in $M$ that join $x$ and $y$. This definition will satisfy all the usual properties of a metric:

(i) $d(x, y) \geq 0$,

(ii) $d(x, y)=d(y, x)$,

(iii) $d(x, y)+d(y, z) \geq d(x, z)$.

DEFINITION 2.3. An isometry is a diffeomorphism $\phi: M \rightarrow N$ that preserves the distance function $d_{N}(\phi(x), \phi(y))=d_{M}(x, y)$.

A group $G$ is said to act isometrically on a manifold $M$ if

(i) each element $g$ in $G$ defines a distance-preserving map from $M$ to itself,

(ii) the composition of two such maps coincides with the group operation $g(h(x))$ $=g h(x)$ for every $x$ in $M$ and for every pair $g$ and $h$ in $G$,

(iii) the identity element of $G$, id, is the identity map on $M$.

(An example to keep in mind with respect to group actions is the group of orthogonal matrices acting on $\mathbb{R}^{n}$.)

DEFINITION 2.4. The orbit of a point $x$ is the set $G(x)=\{y \in M: y=g(x)$ for some $g \in G\}$.

Such an action defines an equivalence relation on $M$, since two orbits are either identical or disjoint. (Suppose, for example, $y$ is in the orbit of $x$. Then for some $g$ in $G, g(x)=y$. Then the orbit of $y$ is contained in the orbit of $x$, since $h(y)=h(g(x))$ for every $h$ in $G$. However, $g^{-1}(g(x))=g^{-1}(y)$, so $x=g^{-1}(y)$, and $x$ is also in the orbit of $y$. So we have containment in both directions, and the orbits must be equal.) These orbits are considered as points in a new space, $M / G$, called the orbit space. If $M$ is a metric space, this orbit space is endowed with a natural metric: the distance between points $G(x)$ and $G(y)$ in $M / G$ is the same as the distance between the two sets $G(x)$ and $G(y)$ in $M$. When $G$ is compact, this distance is zero only when the orbits coincide.

DEFINITION 2.5. In general, for $M$ a Riemannian $n$-manifold, we say that $G$ acts by cohomogeneity $k$ on $M$ when $\operatorname{dim}(M / G)=k$.

For instance, Example 1.1 exhibits a cohomogeneity-one action.

The isometries under consideration are from the sphere to itself. We will show how various orbit spaces are obtained. We will also find which subgroups of $G$-called isotropy subgroups-fix different points in the manifold.

DEFINITION 2.6. When a manifold $M$ is acted on by a group $G$, the isotropy subgroup (or stability subgroup) $G_{x}$ of a point $x$ in the manifold is the subgroup that fixes $x$; that is, $G_{x}=\{g \in G: g(x)=x\}$.

By the definition of a group action, $G_{x}$ always contains the identity element of the group. If two points $x$ and $y$ in $M$ belong to the same orbit, their isotropy subgroups 
are conjugate. If $g(x)=y$, then for any $h \in G_{y}, h(g(x))=h(y)=y$, and $g^{-1}(h g(x))$ $=g^{-1}(y)=x$, so $g^{-1} h g \in G_{x}$. Therefore, $g^{-1} G_{y} g \subset G_{x}$. Since $x=g^{-1}(y), g G_{x} g^{-1} \subset$ $G_{y}$. Since we have containment both ways, $g G_{x} g^{-1}=G_{y}$. Two orbits $G(x)$ and $G(y)$ are of the same type if $G_{x}$ and $G_{y}$ are conjugate in $G$.

DEFINITION 2.7. A principal isotropy subgroup (in terms of dimension, the "smallest") is an isotropy subgroup $H$ of $G$ such that for every other isotropy subgroup $K$, $K \supseteq \mathrm{gHg}^{-1}$ for some $g$ in $G$.

This group (all principal isotropy subgroups are conjugate and hence isomorphic) is associated with a special class of orbits.

DEFINITION 2.8. Orbits having a conjugate of $H$ as their isotropy subgroup are called the principal orbits.

Orbits whose isotropy subgroups are of a strictly higher dimension than $H$ are called singular orbits.

The union of these principal orbits form an open, dense submanifold of $M$, called the regular part of $M[6]$.

In Example 1.1, the principal isotropy subgroup is the identity, because any point in $S^{2}$ without zero entries is not fixed by any nontrivial subgroup of the circle. That is, for $x$ and $y$ nonzero,

$$
\left[\begin{array}{c}
x \cos \theta-y \sin \theta \\
x \sin \theta+y \cos \theta \\
z
\end{array}\right]=\left[\begin{array}{l}
x \\
y \\
z
\end{array}\right]
$$

implies that $\cos \theta=1$. However, at the points $(0,0, \pm 1)$, the isotropy subgroup is all of $S^{1}$, because the whole circle fixes these points. Therefore, these are singular orbits, and their associated isotropy subgroup is $S^{1}$.

Principal orbits form the interior of the orbit space $M / G$, and singular orbits form the boundary.

DEFINITION 2.9. A representation of a group $G$ on a vector space $V$ is homomorphism $\rho$ from $G$ to the group of all invertible linear transformations on $V$; that is, $\rho: G \rightarrow \mathrm{GL}(V)$.

A representation of $G$ on $V$ is called reducible if there is a proper, nontrivial $G^{-}$ invariant subspace of $V$. Otherwise, it is called irreducible.

We wish to study the representations of $G$ into $\operatorname{GL}(V)$ such that $\rho(G)$ acts on $S^{n}$ for some $n$. For the sake of brevity, we often write $g(v)$ for $\rho(g)(v)$, when $\rho$ is clear from the context.

\section{Background and examples}

Definition 3.1. A Lie group $G$ is a group that is also a manifold, on which the group operations define differentiable maps. That is, $\mu: G \times G \rightarrow G$ by $\mu(g, h)=g h$ is a differentiable map, as is $g \rightarrow g^{-1}$. (The circle $S^{1}$ is a Lie group under multiplication: $0 e^{i \theta} e^{i \phi}=e^{i(\theta+\phi)}$.) 
Let $G$ be a compact, connected Lie group, acting isometrically on $S^{n}$, the standard sphere in $\mathbb{R}^{n+1}$ of radius 1 .

As previously mentioned, in Example 1.1, we have an action of cohomogeneity one (since the orbit space is one dimensional). On the other hand, if we allow the entire orthogonal group to act on $S^{n}$, there is only one orbit, since every point $x \in S^{n}$ is the image of the point $(1,0, \ldots, 0)$ under multiplication by a matrix with the vector $x$ in the first column. This is a cohomogeneity-zero action.

Definition 3.2. A geodesic on a manifold $M$ is a parameterized curve $c$ whose acceleration $c^{\prime \prime}$ is always perpendicular to $M$.

EXAMPLE 3.3. One of the classic examples of a group action on a sphere is the Hopf action $S^{1}$ acting on $S^{3}$. This action can be represented as

$$
\left[\begin{array}{cc}
e^{i \theta} & 0 \\
0 & e^{-i \theta}
\end{array}\right]\left[\begin{array}{c}
r e^{i \alpha} \\
s e^{i \beta}
\end{array}\right],
$$

where $r$ and $s$ are nonnegative real numbers with $r^{2}+s^{2}=1$. This is a reducible action; it fixes the geodesics $\left[\begin{array}{c}e^{i \alpha} \\ 0\end{array}\right]$ and $\left[\begin{array}{c}0 \\ e^{i \beta}\end{array}\right]$. By choosing $\theta=-\alpha$, we see that a typical orbit contains a point of the type $\left(r, s e^{i \phi}\right)$; therefore, the orbit space has dimension one less than $S^{3}$ : it is two dimensional. Only the identity fixes a point of this type, so the principal isotropy group is trivial. Moreover, there are no singular orbits, and hence, no edges or vertices.

In the examples below, we find the orbit spaces and the isotropy subgroups associated with these actions through repeated use of the following facts:

(1) constant matrices, that is, $k I_{n}$, where $k$ is a scalar, are in the center of $\operatorname{GL}(n, V)$. This is because multiplication by a scalar matrix is identical to multiplication by the scalar itself as it multiplies every entry in the other matrix by that scalar;

(2) over a commutative field such as $\mathbb{R}$ or $\mathbb{C}$, the only matrices that commute with diagonal matrices having unequal entries along the diagonal are other diagonal matrices;

(3) the two diagonal entries in a diagonal $2 \times 2$ matrix may be interchanged through conjugation by

$$
\left[\begin{array}{cc}
0 & 1 \\
-1 & 0
\end{array}\right]
$$

Consequently, any two diagonal entries in any diagonal matrix may be interchanged through an appropriate conjugation by elementary matrices.

One method of determining the orbit decomposition of a cohomogeneity-two action by a group $G$ is to rely on the classification of cohomogeneity-one actions 
(cf. $[3,4,8,9,10,11]$ ). We note that when $S^{n} / G=D^{2}$, the singular orbits form the boundary of the disk and the singular isotropy subgroups at those points act on the unit normal subspace to the orbits by cohomogeneity one. Since the cohomogeneityone actions have been classified, it suffices to find the singular isotropy subgroups $K$ with $H \subset K \subset G$ that act on the normal subspace by cohomogeneity one.

Another method entails looking at the matrix representation of $G$ in the group of $n \times n$ orthogonal matrices $\mathrm{O}(n)$. We then take a "typical" vector in $S^{n}$, and try to see how $G$ identifies that vector with other vectors. Basically, we try to reduce the dimensions in the $(n+1)$-vector by replacing as many entries as possible by zero, and still remain within the same orbit. Then, we use this simplified vector to see what subgroup of $G$ would fix that vector. In this way, we find the principal isotropy subgroup. We look at less typical vectors, ones with more zeros, perhaps, or more identifications among the entries, to find other possible isotropy subgroups. These computations give a very explicit description of the elements in the orbit space as subspaces in $\mathbb{R}^{n}$, and any edges and vertices. Sometimes, however, it is very difficult to write out an explicit matrix description of the group action. Therefore, we need the two methods to complement one another.

4. Reducible examples. A whole body of very simple reducible examples is obtained by adding either a one-dimensional trivial action (1) or a transitive action by $\mathrm{SO}(k)$ to cohomogeneity-one actions on spheres. In this section, we will look at the reducible actions $(G, \phi)=\left(\mathrm{SO}\left(n_{1}\right) \times \mathrm{SO}\left(n_{2}\right) \times \mathrm{SO}\left(n_{3}\right), \rho_{n_{1}}+\rho_{n_{2}}+\rho_{n_{3}}\right)$, where $\rho_{n}$ is the standard representation of $\mathrm{SO}(n),(G, \phi)=\left(\mathrm{SO}(2) \times \mathrm{SO}(m)(m>2), \rho_{2} \otimes \rho_{m}+1\right)$, where 1 is a one-dimensional trivial representation, and $(G, \phi)=\left(\mathrm{SO}(k), 2 \rho_{k}\right)$, for $k \geq 3$. We will describe the resulting orbit spaces under these actions.

EXAMPLE 4.1. The action of $\mathrm{SO}\left(n_{1}\right) \times \mathrm{SO}\left(n_{2}\right) \times \mathrm{SO}\left(n_{3}\right)$ on $S^{n_{1}+n_{2}+n_{3}-1}$.

If we add a transitive action by $\mathrm{SO}(n)$ to a cohomogeneity-one action, as in $(G, \phi)=$ $\left(\mathrm{SO}\left(n_{1}\right) \times \mathrm{SO}\left(n_{2}\right) \times \mathrm{SO}\left(n_{3}\right), \rho_{n_{1}}+\rho_{n_{2}}+\rho_{n_{3}}\right)$, we add another dimension to our orbit space. Under this action, vectors in $\mathbb{R}^{n_{1}+n_{2}+n_{3}}$ are acted on by matrices of the type

$$
\left[\begin{array}{lll}
A & & \\
& B & \\
& & C
\end{array}\right]
$$

where $A$ is in $\mathrm{SO}\left(n_{1}\right), B$ is in $\mathrm{SO}\left(n_{2}\right)$, and $C$ is in $\mathrm{SO}\left(n_{3}\right)$. Each matrix may be taken to reduce the vector of the appropriate dimension to its length. Think of the appropriate vector in $\mathbb{R}^{n_{1}+n_{2}+n_{3}}$ as the direct sum of $\mathbf{v}_{\mathbf{1}} \in \mathbb{R}^{n_{1}}, \mathbf{v}_{\mathbf{2}} \in \mathbb{R}^{n_{2}}$, and $\mathbf{v}_{\mathbf{3}} \in \mathbb{R}^{n_{3}}$; that is, $\left(\left(\mathbf{v}_{1}\right),\left(\mathbf{v}_{2}\right),\left(\mathbf{v}_{3}\right)\right)$. Then, by choosing the first row in $A$ to be a unit vector parallel to $\mathbf{v}_{\mathbf{1}}$, the first $n_{1}$ entries of the image are reduced to $\left(\left\|\mathbf{v}_{\mathbf{1}}\right\|, 0, \ldots, 0\right)$. Similarly, $B$ and $C$ can be chosen to reduce $\mathbf{v}_{2}$ and $\mathbf{v}_{3}$ to their lengths. Thus, every orbit space contains an element of the form $(x, 0, \ldots, 0, y, 0, \ldots, 0, z, 0, \ldots, 0)$, where $x, y$, and $z$ are all nonnegative. As the three direct summands do not interact with each other, there are no further identifications. (We cannot interchange the $x$ and $y$, for instance.) 
Therefore, the principal isotropy group is isomorphic to $\mathrm{SO}\left(n_{1}-1\right) \times \mathrm{SO}\left(n_{2}-1\right) \times$ $\mathrm{SO}\left(n_{3}-1\right)$, in a form conjugate to

$$
\left[\begin{array}{llllll}
1 & & & & & \\
& A^{\prime} & & & & \\
& & 1 & & & \\
& & & B^{\prime} & & \\
& & & & 1 & \\
& & & & & C^{\prime}
\end{array}\right],
$$

where $A^{\prime}$ is an element of $\mathrm{SO}\left(n_{1}-1\right), B^{\prime}$ is an element of $\mathrm{SO}\left(n_{2}-1\right)$, and $C^{\prime}$ is an element of $\mathrm{SO}\left(n_{3}-1\right)$. The orbit space may be described as the triangle in the twosphere that inhabits the first octant: $\left\{(x, y, z) \in S^{2}: x \geq 0 ; y \geq 0 ; z \geq 0\right\}$. The edges are $x=0, y=0$, and $z=0$. On these edges, the submatrix that corresponds to the subspace of the zero vector acts trivially, so the isotropy subgroup on the edge corresponding to the $n_{i}$-dimensional vector has a factor of $\mathrm{SO}\left(n_{i}\right)$ replacing the factor $\mathrm{SO}\left(n_{i}-1\right), \mathrm{SO}\left(n_{1}\right) \times \mathrm{SO}\left(n_{2}-1\right) \times \mathrm{SO}\left(n_{3}-1\right)$ is the isotropy subgroup of points for which $\mathbf{v}_{\mathbf{1}}=\mathbf{0}$, for instance. At the vertices, $(1,0,0),(0,1,0)$, and $(0,0,1)$, two of the $\mathrm{SO}\left(n_{i}-1\right)$ factors are replaced by $\mathrm{SO}\left(n_{i}\right)$. We have now found the orbit space and all the isotropy subgroups. The decomposition of this action is given in Figure 4.1.

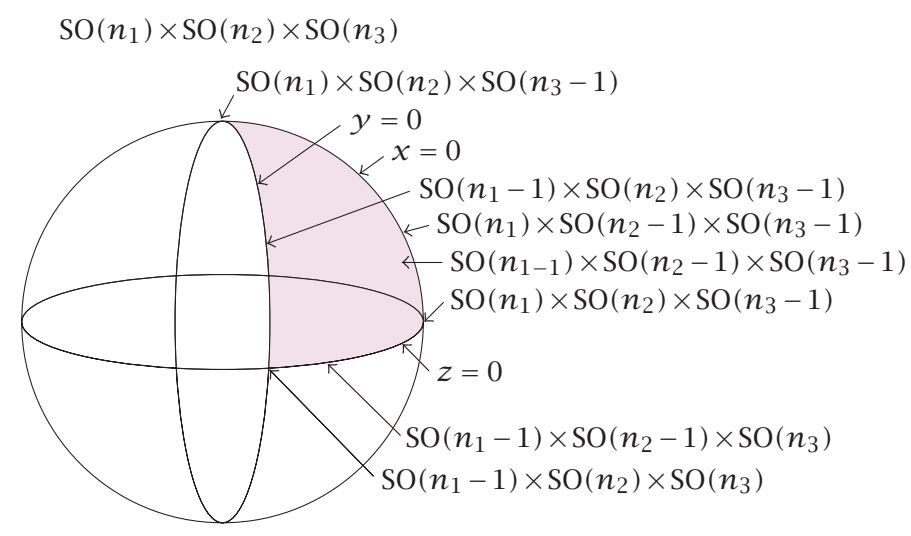

FIGURE 4.1

EXAMPLE 4.2. The action of $\mathrm{SO}(2) \times \mathrm{SO}(m)$ on $S^{2 m}$.

Suppose, instead, that we add a trivial action to a cohomogeneity-one action. For example, take $(G, \phi)=\left(\mathrm{SO}(2) \times \mathrm{SO}(m), \rho_{2} \otimes \rho_{m}+1\right)$. Under this action, the first $2 m$ entries in the $(2 m+1)$-vector in $S^{2 m}$ are represented as a $2 \times m$ matrix. This matrix is acted on by left multiplication by elements of $\mathrm{SO}(2)$ and by right multiplication by elements of $\mathrm{SO}(m)$. The last entry of the $(2 m+1)$-vector in $S^{2 m}$ is the object of the trivial action; that is, it is left unchanged. The right and left actions by $\mathrm{SO}(2)$ and 
$\mathrm{SO}(m)$, respectively, can serve to "diagonalize" the $2 \times m$ matrix, that is, to leave all the entries zero save $a_{11}$ and $a_{22}$. (This is a standard theorem of linear algebra. See, e.g., [7, page 179].) Moreover, we may assume both entries to be positive, since either may be made positive by multiplication by an appropriate element of $\mathrm{SO}(m)$. As we have noted in (3), we can interchange $x$ and $y$, so we may assume that $x \geq y \geq 0$. Thus, the orbit space is the subset of the two-sphere given by $\left\{(x, y, z) \in S^{2}: x \geq y \geq 0\right\}$. It is the spherical lune cut by the planes $x=y$ and $y=0$. Along the edge $x=y$, the isotropy subgroup is isomorphic to $\mathrm{SO}(2) \times \mathrm{SO}(m-2)$, in a form conjugate to $\left\{B \otimes\left[B^{-1}{ }_{A}\right]: B \in \mathrm{SO}(2) ; A \in \mathrm{SO}(m-2)\right\}$.

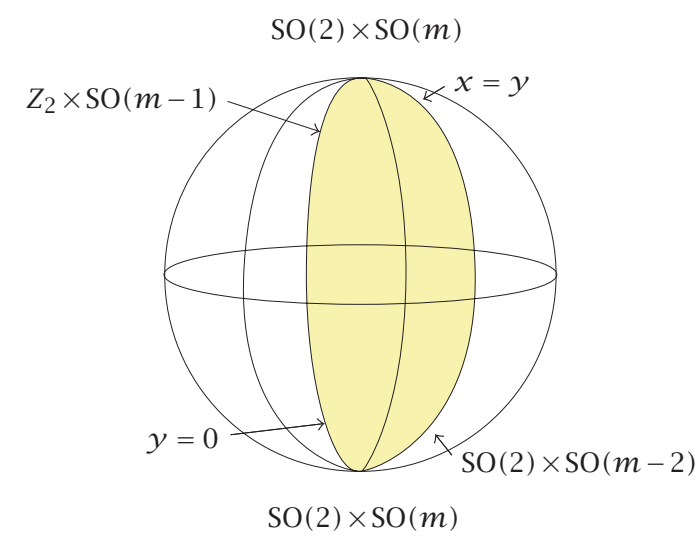

FIGURE 4.2

Along the edge $y=0$, the isotropy subgroup is isomorphic to $\mathbb{Z}_{2} \times \mathrm{SO}(m-1)$, in a form conjugate to $\left\{ \pm I_{2} \otimes\left[{ }_{A}^{ \pm 1}\right]: A \in \mathrm{O}(m-1)\right\}$. (This group is also denoted $S(\mathrm{O}(1) \mathrm{O}(m-1))$.) For a fixed value of $z$, the decomposition of the orbit space is exactly the same as it is for the cohomogeneity-one action. Thus, the decomposition of the cohomogeneity-two action may be thought of as a suspension of the cohomogeneity-one action along each level $z=c$ between the poles. The poles, $z= \pm 1$, are fixed points under the action. In Figure 4.2, there is a diagram of the orbit space and the isotropy decomposition for the cohomogeneity-two action.

EXAMPLE 4.3. The action of $(G, \phi)=\left(\mathrm{SO}(k), 2 \rho_{k}\right)$ on $S^{2 k-1}$.

Finally, we examine a reducible cohomogeneity-two action that is not a direct sum with a cohomogeneity-one action: $(G, \phi)=\left(\mathrm{SO}(k), 2 \rho_{k}\right)$, for $k \geq 3$. Under this action, $\mathrm{SO}(k)$ acts on $\mathbb{R}^{2 k}$ by matrices of the form

$$
\left[\begin{array}{ll}
A & \\
& A
\end{array}\right],
$$

where $A$ is in $\mathrm{SO}(k)$. We can think of the first $k$ entries of the vector in $\mathbb{R}^{2 k}$ as one vector, $\mathbf{v}_{\mathbf{1}}$, in $\mathbb{R}^{k}$, and the second $k$ entries as another, $\mathbf{v}_{\mathbf{2}}$. If these two vectors are linearly independent, they span a plane. If we choose $A$ to be a matrix in $\mathrm{SO}(k)$ whose first two rows span the same plane, with the first row being parallel to the first $k$ vector, and the second row resulting in the same orientation for the plane as the pair 


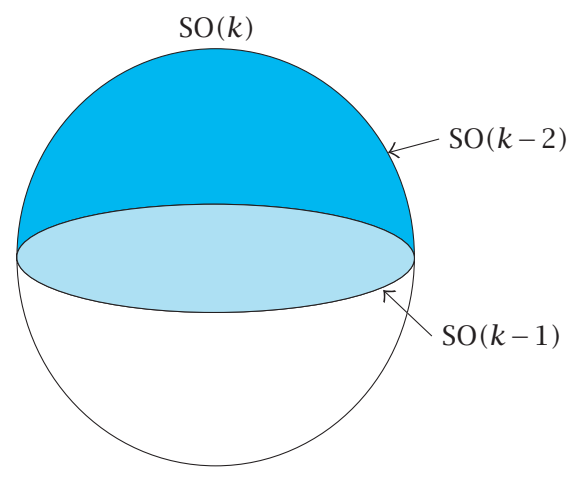

FIGURE 4.3

of $k$-vectors, the image of the $\mathbb{R}^{2 k}$ vector under multiplication by this matrix will be a vector of the form $(x, 0, \ldots, 0 ; y, z, 0, \ldots, 0)$, with $x \geq 0$, and $z \geq 0$. (The semicolon signifies the end of the first $k$-vector.) The principal isotropy subgroup will therefore be a conjugate of the group of matrices of the form

$$
\left[\begin{array}{llll}
I_{2} & & & \\
& B & & \\
& & I_{2} & \\
& & & B
\end{array}\right],
$$

where $B$ is in $\mathrm{SO}(k-2)$. We have larger isotropy subgroups when rank $\left(\mathbf{v}_{\mathbf{1}}, \mathbf{v}_{2}\right)=1$. Then, either $\mathbf{v}_{\mathbf{1}}$ is parallel to $\mathbf{v}_{\mathbf{2}}$ or one of the vectors is zero. If they are parallel, the orbit has an element of the form $(x, 0, \ldots, 0 ; y, 0, \ldots, 0)$, and the isotropy subgroup is isomorphic to $\mathrm{SO}(k-1)$. The isotropy subgroup is also $\mathrm{SO}(k-1)$ if either $\mathbf{v}_{\mathbf{1}}=\mathbf{0}$ or $\mathbf{v}_{\mathbf{2}}=\mathbf{0}$, because the orbit representative is the same, except that either $x$ or $y$ is zero. The orbit space is therefore a disk without vertices. In fact, it is the upper hemisphere of the sphere of radius $1 / 2$. We can see this by inspecting the geodesics of the orbit space. The boundary geodesic is $(\cos \theta, 0, \ldots, 0 ; \sin \theta, 0, \ldots, 0)$. This geodesic closes at $\theta=\pi$, since $x=1$ and $x=-1$ are identified; it reaches its most distant position at $\theta=\pi / 2$. Longitudinal geodesics may be described as $(\cos \alpha \sin \phi, 0, \ldots, 0 ; \sin \alpha \sin \phi, \cos \phi, 0, \ldots, 0)$, where $\alpha \in[0, \pi)$ is fixed, and $\phi \in$ $[-\pi / 2, \pi / 2)$. As mentioned above, $(x, 0, \ldots, 0 ; y, 0, \ldots, 0)$ and $(-x, 0, \ldots, 0 ;-y, 0, \ldots, 0)$ are identified under the group action, so the geodesic closes there. The piece of the geodesic that lies in the orbit space is that with $\cos \phi>0$. This is half the geodesic; it has length $\pi / 2$. These geodesics are perpendicular to the underlying orbits, because no element of the group action changes the lengths of $\mathbf{v}_{\mathbf{1}}$ or $\mathbf{v}_{\mathbf{2}}$, and the geodesic moves strictly in the direction that would change these lengths. The restriction $z \geq 0$ makes this orbit space the upper hemisphere. The orbit space diagram is given in Figure 4.3.

The resulting orbit spaces for the reducible cohomogeneity-two actions on $S^{n}$ above are given in Table 4.1 .

Here, we label a spherical lune with both angles $\pi / n$ as $D_{n}$, a spherical triangle in the unit two-sphere with angles $\pi / k, \pi / n$, and $\pi / m$ as $\Delta_{k, n, m}$, and the upper 
TABLE 4.1

\begin{tabular}{lll}
\hline Group & Representation & Orbit space \\
\hline $\mathrm{SO}\left(n_{1}\right) \times \mathrm{SO}\left(n_{2}\right) \times \mathrm{SO}\left(n_{3}\right)$ & $\rho_{n_{1}}+\rho_{n_{2}}+\rho_{n_{3}}$ & $\Delta_{2,2,2}$ \\
$\mathrm{SO}(2) \times \mathrm{SO}(m)$ & $\rho_{2} \otimes \rho_{m}+1$ & $D_{4}$ \\
$\mathrm{SO}(k)$ & $2 \rho_{k}$ & $(1 / 2) S^{2}(1 / 2)$ \\
\hline
\end{tabular}

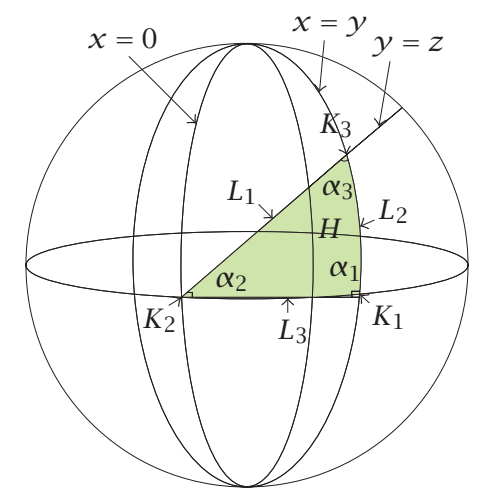

FiguRE 4.4

TABLE 4.2. Reducible cohomogeneity-two orbit spaces.

\begin{tabular}{|c|c|c|}
\hline Group & \multicolumn{2}{|c|}{$\mathrm{SO}\left(n_{1}\right) \times \mathrm{SO}\left(n_{2}\right) \times \mathrm{SO}\left(n_{3}\right)$} \\
\hline Representation & \multicolumn{2}{|c|}{$\rho_{n_{1}}+\rho_{n_{2}}+\rho_{n_{3}}$} \\
\hline Angles & \multicolumn{2}{|c|}{$\alpha_{1}=\pi / 2, \alpha_{2}=\pi / 2, \alpha_{3}=\pi / 2$} \\
\hline$H$ & & $\mathrm{SO}\left(n_{1}-1\right) \times \mathrm{SO}\left(n_{2}-1\right) \times \mathrm{SO}\left(n_{3}-1\right)$ \\
\hline$L_{1}$ & & $\mathrm{SO}\left(n_{1}-1\right) \times \mathrm{SO}\left(n_{2}-1\right) \times \mathrm{SO}\left(n_{3}\right)$ \\
\hline$L_{2}$ & & $\mathrm{SO}\left(n_{1}\right) \times \mathrm{SO}\left(n_{2}-1\right) \times \mathrm{SO}\left(n_{3}-1\right)$ \\
\hline$L_{3}$ & & $\mathrm{SO}\left(n_{1}-1\right) \times \mathrm{SO}\left(n_{2}\right) \times \mathrm{SO}\left(n_{3}-1\right)$ \\
\hline$K_{1}$ & & $\mathrm{SO}\left(n_{1}-1\right) \times \mathrm{SO}\left(n_{2}\right) \times \mathrm{SO}\left(n_{3}\right)$ \\
\hline$K_{2}$ & & $\mathrm{SO}\left(n_{1}\right) \times \mathrm{SO}\left(n_{2}-1\right) \times \mathrm{SO}\left(n_{3}\right)$ \\
\hline$K_{3}$ & & $\mathrm{SO}\left(n_{1}\right) \times \mathrm{SO}\left(n_{2}\right) \times \mathrm{SO}\left(n_{3}-1\right)$ \\
\hline Group & $\mathrm{SO}(2) \times \mathrm{SO}(m)$ & $\mathrm{SO}(k)$ \\
\hline Representation & $\rho_{2} \otimes \rho_{m}+1$ & $2 \rho_{k}$ \\
\hline $\begin{array}{l}\text { Angles } \\
H\end{array}$ & $\begin{array}{l}\alpha_{1}=\pi / 4, \alpha_{2}=\pi / 4, \alpha_{3}=\pi \\
\mathbb{Z}_{2} \times \operatorname{SO}(m-2)\end{array}$ & $\begin{array}{l}\alpha_{1}=\pi, \alpha_{2}=\pi, \alpha_{3}=\pi \\
\mathrm{SO}(k-2)\end{array}$ \\
\hline$L_{1}$ & $\mathbb{Z}_{2} \times \mathrm{SO}(m-1)$ & $\mathrm{SO}(k-1)$ \\
\hline$L_{2}$ & $\mathrm{SO}(2) \times \mathrm{SO}(m-2)$ & - \\
\hline$L_{3}$ & - & - \\
\hline$K_{1}$ & $\mathrm{SO}(2) \times \mathrm{SO}(m)$ & - \\
\hline$K_{2}$ & $\mathrm{SO}(2) \times \mathrm{SO}(m)$ & - \\
\hline$K_{3}$ & - & - \\
\hline
\end{tabular}


hemisphere of the sphere of radius $r$ as $(1 / 2) S^{2}(r)$. The third orbit space is computed and described in both $[4,8]$; the other two may be readily seen by reference to the cohomogeneity-one actions described in $[4,9,10]$.

Moreover, they have decompositions as given in Table 4.2, where $H, L_{i}$, and $K_{i}$ are the isotropy subgroups associated with the orbit space as in Figure 4.4. The principal isotropy groups here are also presented in $[4,9,10]$. The others may be computed easily with reference to $[9,10]$.

5. Irreducible examples. In this section, we will examine the following irreducible actions: $(G, \phi)=\left(\mathrm{SO}(3) \times \mathrm{SO}(n), \rho_{3} \otimes \rho_{n}\right)$ on $S^{3 n-1},(G, \phi)=\left(\mathrm{SO}(3) \times \mathrm{SO}(3), \rho_{3} \otimes \rho_{3}\right)$ on $S^{8},(G, \phi)=\left(\mathrm{U}(3) \times \mathrm{SU}(n), \rho_{2} \times \mu_{3} \otimes \mu_{n}\right)$ on $S^{6 n-1}$, and $(G, \phi)=\left(\operatorname{Sp}(3) \times \operatorname{Sp}(n), v_{3} \otimes v_{n}\right)$ on $S^{12 n-1}$. Each of these examples relies on the following fact from linear algebra: an arbitrary real (complex, quaternion) $n \times m$ matrix may be "diagonalized" under left multiplication by an $n \times n$ orthogonal (unitary, symplectic) matrix and right multiplication by an $m \times m$ orthogonal (unitary, symplectic) matrix. By "diagonalized," we mean that the only nonzero terms are among the entries $a_{i i}$. (See [7, page 179].) All four of the following group actions result in spherical triangles as orbit spaces, so rather than include a figure for each one, we have included one figure at the end of the section to describe them all.

EXAMPLE 5.1. The action of $\mathrm{SO}(3) \times \mathrm{SO}(n), n>3$, on $S^{3 n-1}$.

One action that generates a triangular orbit space is $(G, \phi)=\left(\mathrm{SO}(3) \times \mathrm{SO}(n), \rho_{3} \otimes\right.$ $\rho_{n}$ ), where $n>3$. Under this action, points of $S^{3 n-1}$ are represented as $3 \times n$ matrices, operated on by $\mathrm{SO}(3)$ under left multiplication, and by $\mathrm{SO}(n)$ by right multiplication. Appropriate choices for the left and right multipliers will reduce the middle matrix $(X)$ to one of the form

$$
\left[\begin{array}{lllll}
a & 0 & 0 & 0 & \cdots \\
0 & b & 0 & 0 & \cdots \\
0 & 0 & c & 0 & \cdots
\end{array}\right]
$$

where $a^{2}, b^{2}$, and $c^{2}$ are eigenvalues of $X X^{T}$.

These matrix actions render the signs of $a, b$, and $c$ immaterial and allow for shifts in the order of these entries so that $a \geq b \geq c \geq 0$. Since $a^{2}, b^{2}$, and $c^{2}$ are eigenvalues of $X X^{T}$, no further identifications are possible. The orbit space is therefore the spherical triangle $\left\{(x, y, z) \in S^{2}: x \geq y \geq z \geq 0\right\}$. Since a diagonal matrix is fixed under conjugation by other diagonal matrices, the principal isotropy group is conjugate to

$$
\left\{A \otimes\left[\begin{array}{cc}
A^{-1} & 0 \\
0 & B
\end{array}\right], A \in \mathrm{SO}(3), A \text { a diagonal matrix, } B \in \mathrm{O}(n-3)\right\} .
$$

The fact that $A$ is diagonal and in $\mathrm{SO}(3)$ forces it to have entries equal to \pm 1 on its diagonal (the number of minus signs being even). Therefore, the principal isotropy group is isomorphic to $S(\mathrm{O}(1) \mathrm{O}(1) \mathrm{O}(1) \mathrm{O}(n-3))$. 
On one edge of the spherical triangle, where $z=0$, the points will be fixed under multiplication by group elements of the form

$$
\left\{\left[\begin{array}{cc}
A & 0 \\
0 & \pm 1
\end{array}\right] \otimes\left[\begin{array}{cc}
A^{-1} & 0 \\
0 & B
\end{array}\right], A \in \mathrm{O}(2), A \text { a diagonal matrix, } B \in \mathrm{O}(n-2)\right\}
$$

That is, the upper block matrix, which conjugates the nonzero entries in $X$, can be shrunk to a $2 \times 2$ block, since the $z$ entry is now zero. The isotropy subgroup is therefore $S(\mathrm{O}(1) \mathrm{O}(1) \mathrm{O}(n-2))$.

On the edges where $x=y$ or $y=z$, the $2 \times 2$ constant matrix is preserved by conjugation by block matrices in $\mathrm{O}(2)$. The third (unequal) entry can still be conjugated by -1 . The isotropy subgroup on these edges is therefore isomorphic to $S(\mathrm{O}(2) \mathrm{O}(1) \mathrm{O}(n-3))$.

The orbit space, which is a spherical triangle, has angles $\pi / 2, \pi / 3$, and $\pi / 4$. The isotropy subgroup at $y=z=0$ is isomorphic to $\mathbb{Z}_{2} \times \mathrm{SO}(2) \times \mathrm{SO}(n-1)$, since the zeroblock matrix will be unaffected by any matrix multiplication. The isotropy subgroup at the intersection of $x=y$ and $z=0$ is isomorphic to $\mathrm{SO}(2) \times \mathbb{Z}_{2} \times \mathrm{SO}(n-2)$, in a form conjugate to

$$
\left\{\left[\begin{array}{cc}
A & 0 \\
0 & \pm 1
\end{array}\right] \otimes\left[\begin{array}{cc}
A^{-1} & 0 \\
0 & B
\end{array}\right], A \in \mathrm{O}(2), B \in \mathrm{O}(n-2)\right\}
$$

since the $2 \times 2$ block matrix with equal entries on the diagonal will commute with any $2 \times 2$ matrix. At the point where $x=y=z$, the isotropy subgroup is isomorphic to $S(\mathrm{O}(3) \mathrm{O}(n-3))$, since at this vertex, the $3 \times 3$ scalar block of the matrix $X$ is fixed under conjugation by any element of $\mathrm{O}(3)$.

EXAMPLE 5.2. The action of $\mathrm{SO}(3) \times \mathrm{SO}(3)$ on $S^{8}$.

A related action, but with a different orbit space, $(G, \phi)=\left(\mathrm{SO}(3) \times \mathrm{SO}(3), \rho_{3} \otimes \rho_{3}\right)$, can again reduce the spherical $3 \times 3$ matrix $(X)$ to diagonal form, where the squares of the diagonal entries are the eigenvalues of $X X^{T}$. As before, we can interchange any pair of these entries. However, we can no longer change the sign of a single entry, but must change them two at a time, since we no longer have the freedom to insert a -1 entry below the upper $3 \times 3$ matrix in the right factor. Thus, we can ensure only that the entries are in order of descending absolute value and that the first two are positive: $\left\{(x, y, z) \in S^{2}: x \geq y \geq|z|\right\}$. This triangle is the double of the triangle under the $\mathrm{SO}(3) \times \mathrm{SO}(n)$ action, reflected through the plane $z=0$. The edges of this triangle are the intersections of the two-sphere with the planes $x=y, y=z$, and $y=-z$.

The principal isotropy group must fix diagonal $3 \times 3$ matrices. As we saw above, only conjugation by diagonal matrices will fix these; the diagonal matrices in $\mathrm{SO}(3)$ are those with \pm 1 on the diagonal (with the number of -1 's being even). Hence, the principal isotropy subgroup is $S(\mathrm{O}(1) \mathrm{O}(1) \mathrm{O}(1))$. On the edges $x=y$ and $y=z$, we can clearly conjugate the $2 \times 2$ constant matrix by any element of $\mathrm{O}(2)$; therefore, the 
isotropy subgroups on these two edges are $S(\mathrm{O}(2) \mathrm{O}(1))$ and $S(\mathrm{O}(1) \mathrm{O}(2))$, respectively. Moreover, we get an isomorphic group on the edge $y=-z$

$$
\left[\begin{array}{ll} 
\pm 1 & \\
& A
\end{array}\right]\left[\begin{array}{ll}
I_{2} & \\
& -1
\end{array}\right]\left[\begin{array}{ccc}
x & 0 & 0 \\
0 & y & 0 \\
0 & 0 & -y
\end{array}\right]\left[\begin{array}{ll} 
\pm 1 & \\
& A^{-1}
\end{array}\right]\left[\begin{array}{cc}
I_{2} & \\
& -1
\end{array}\right],
$$

where $A \in \mathrm{SO}$ (2) if the \pm 1 in the upper-right corner is negative, and $A \in \mathrm{O}$ (2) with determinant -1 , if the sign of the 1 is positive. (Thus, the product of the first two factors is in $\mathrm{SO}(3)$.) The isotropy subgroups on the vertices are computed analogously with those in the previous action, except in the case of the third vertex, where we again multiply on both sides by $\left[\begin{array}{ll}I_{2} & \\ & -1\end{array}\right]$ to make the most use of the symmetries.

EXAMPLE 5.3. The action of $\mathrm{U}(3) \times \mathrm{SU}(n)$ on $S^{6 n-1}$.

We can apply a similar action with the unitary group $(G, \phi)=\left(\mathrm{U}(3) \times \mathrm{SU}(n),\left(\rho_{2} \times\right.\right.$ $\left.\left.\mu_{3}\right) \otimes \mu_{n}\right)$. The unitary group action behaves very similarly to the real action: the left and right multiplication can reduce the $3 \times n$ matrix $X$ to the three nonzero entries

$$
\left[\begin{array}{ccccc}
x & 0 & 0 & 0 & \cdots \\
0 & y & 0 & 0 & \cdots \\
0 & 0 & z & 0 & \cdots
\end{array}\right]
$$

The squares of these entries are the eigenvalues of $X \bar{X}^{T}$, which are real because $X \bar{X}^{T}$ is Hermitian. Therefore, the entries $x, y$, and $z$ are either real or purely imaginary; they can be made real by multiplication by an appropriate element of U(3). They can be arranged in order of descending absolute value, and, because the first factor group is $\mathrm{U}(3)$, they can all be made positive; the orbit space is

$$
\left\{(x, y, z) \in S^{2}: x \geq y \geq z \geq 0\right\} .
$$

The principal isotropy subgroup is $S\left(\mathrm{U}(1)^{3} \mathrm{U}(n-3)\right)$, as any of the entries, $x, y$, or $z$, can be conjugated by $e^{i \theta}$. On the edges and vertices, the isotropy subgroups are analogous to those under the $\mathrm{SO}(3) \times \mathrm{SO}(n)$ action.

EXAMPLE 5.4. The action of $\operatorname{Sp}(3) \times \operatorname{Sp}(n)$ on $S^{12 n-1}$.

The action by the symplectic group $(G, \phi)=\left(\operatorname{Sp}(3) \times \operatorname{Sp}(n), v_{3} \otimes v_{n}\right)$, where $\operatorname{Sp}(n)$ is the group of Hermitian matrices $\left(A^{*}=A^{-1}\right)$ with quaternion entries, is similar. We note that an $n \times m$ quaternionic matrix can be represented as a $2 n \times 2 m$ complex matrix of the form $\left[\begin{array}{cc}A & -\bar{B} \\ B & \bar{A}\end{array}\right]$. (See, e.g., [2].) A symplectic matrix can be represented by a unitary matrix of this same form. Hence, we are considering an action on $6 \times 2 n$ complex matrices of the form above under left multiplication by a $6 \times 6$ matrix of that form and right multiplication by a $2 n \times 2 n$ matrix of the same form. Now, we know that a complex $6 \times 2 n$ matrix $(X)$ can be "diagonalized" in the sense of diagonalizing a $6 \times 6$ submatrix and leaving all other entries zero, but we need to know whether this can be accomplished by this subgroup of $\mathrm{SU}(6) \times \mathrm{SU}(2 n)$. The answer is yes, because 
TABLE 5.1

\begin{tabular}{lll}
\hline Group & Representation & Orbit space \\
\hline $\mathrm{SO}(3) \times \mathrm{SO}(n)$ & $\rho_{3} \otimes \rho_{n}$ & $\Delta_{2,3,4}$ \\
$\mathrm{SO}(3) \times \mathrm{SO}(3)$ & $\rho_{3} \otimes \rho_{3}$ & $\Delta_{2,3,3}$ \\
$\mathrm{U}(3) \times \mathrm{SU}(3)$ & $\rho_{2} \times \mu_{3} \otimes \mu_{n}$ & $\Delta_{2,3,4}$ \\
$\mathrm{Sp}(3) \times \mathrm{Sp}(n)$ & $\nu_{3} \otimes v_{n}$ & $\Delta_{2,3,4}$ \\
\hline
\end{tabular}

we use the unitary matrix that would diagonalize $\bar{X}^{T} X$, which is a $2 n \times 2 n$ complex matrix of the form $\left[\begin{array}{cc}A & -\bar{B} \\ B & \bar{A}\end{array}\right]$. For any matrix of this form, if $((\mathbf{u}),(\mathbf{v}))$ is an eigenvector with eigenvalue $\lambda$, then $((-\overline{\mathbf{v}}),(\overline{\mathbf{u}}))$ is also an eigenvector, with eigenvalue $\bar{\lambda}$. Since the unitary matrix that diagonalizes $\bar{X}^{T} X$ by conjugation has the eigenvectors of $\bar{X}^{T} X$ as its columns, it, too, can be written in the desired form. Therefore, there exists a $2 n \times 2 n$ unitary matrix $U_{2}$ of the desired form that diagonalizes $\bar{X}^{T} X$.

$$
U_{2} \bar{X}^{T} X U_{2}^{-1}=\left[\begin{array}{cccc}
\beta_{1} & 0 & \cdots & 0 \\
0 & \beta_{2} & \cdots & 0 \\
& & \ddots & \\
0 & \cdots & 0 & \beta_{2 n}
\end{array}\right] \text {. }
$$

The diagonal entries in the resulting matrix $\left(\beta_{i}\right)$ are the eigenvalues of $\bar{X}^{T} X$; they are therefore real and nonnegative. If the column vectors of $X U_{2}^{-1}$ are $\mathbf{v}_{\mathbf{1}}, \mathbf{v}_{2}, \ldots, \mathbf{v}_{2} \mathbf{n}$, equation (5.8) shows that $\left\langle\mathbf{v}_{\mathbf{i}}, \mathbf{v}_{\mathbf{j}}\right\rangle=\beta_{i} \delta_{i j}$. At least $2 n-6$ of the $\beta_{i}$ are zero. Since the eigenvalues of $\bar{X}^{T} X$ come in $\lambda, \bar{\lambda}$ pairs, there is an even number of zeros. Since $X$ and $U_{2}$ are both in the subgroup of complex matrices that represent quaternionic matrices, their product is, so the zeros are evenly distributed in the upper and lower matrices. Therefore, for $\beta_{i}$ nonzero, the $\mathbf{v}_{\mathbf{i}} / \sqrt{\beta_{i}}$ form an orthonormal system, and we can let $U_{1}$ be a unitary matrix with $\mathbf{v}_{\mathbf{i}}^{T} / \sqrt{\beta_{i}}$ as its $i$ th row for each nonzero $\beta$. Then $U_{1} X U_{2}^{-1}$ is a diagonal matrix, and $U_{1}$ and $U_{2}$ are symplectic matrices. The diagonal entries are $\lambda_{1}, \lambda_{2}$, and $\lambda_{3}$ and their conjugates. Since the $\lambda_{i}$ are real, they are equal to their conjugates. We may assume that they are positive and arranged in descending order. Therefore, the orbit space is

$$
\left\{(x, y, z) \in S^{2}: x \geq y \geq z \geq 0\right\}
$$

The isotropy subgroups may be more easily determined by reference to the quaternionic form of the matrices if we remember that the center of the quaternionic group is the reals. Then the reasoning runs analogously to that for $\mathrm{U}(3) \times \mathrm{SU}(n)$.

Therefore, we see that the resulting orbit spaces for these four irreducible cohomogeneity-two actions on $S^{n}$ are given in Table 5.1.

Moreover, they have decompositions as given in the following table, where $H, L_{i}$, and $K_{i}$ are the isotropy subgroups associated with the orbit space as in the following diagram (Figure 5.1 and Table 5.2). 


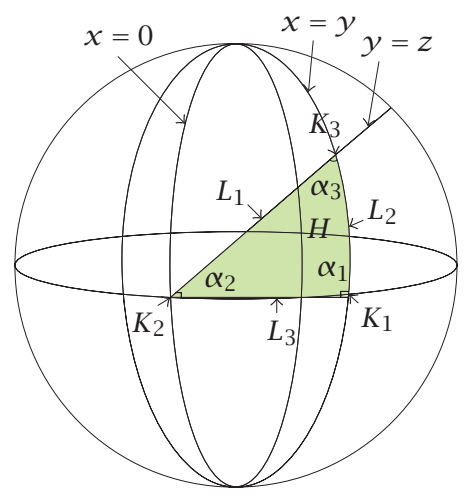

FIGURE 5.1

TABLE 5.2. Irreducible cohomogeneity-two actions.

\begin{tabular}{lll}
\hline Group & $\mathrm{SO}(3) \times \mathrm{SO}(3)$ & $\mathrm{SO}(3) \times \mathrm{SO}(3)$ \\
\hline Representation & $\rho_{3} \otimes \rho_{n}$ & $\rho_{3} \otimes \rho_{3}$ \\
Angles & $\alpha_{1}=\pi / 2, \alpha_{2}=\pi / 4, \alpha_{3}=\pi / 3$ & $\alpha_{1}=\pi / 3, \alpha_{2}=\pi / 2, \alpha_{3}=\pi / 3$ \\
$H$ & $S\left(\mathrm{O}(1)^{2} \times \mathrm{O}(n-3)\right)$ & $S\left(\mathrm{O}(1)^{3}\right)$ \\
$L_{1}$ & $S(\mathrm{O}(1) \times \mathrm{O}(2) \times \mathrm{O}(n-3))$ & $S(\mathrm{O}(1) \times \mathrm{O}(2))$ \\
$L_{2}$ & $S(\mathrm{O}(2) \times \mathrm{O}(1) \times \mathrm{O}(n-3))$ & $S(\mathrm{O}(2) \times \mathrm{O}(1))$ \\
$L_{3}$ & $S\left(\mathrm{O}(1)^{2} \times \mathrm{O}(n-2)\right)$ & $S(\mathrm{O}(1) \times \mathrm{O}(2))$ \\
$K_{1}$ & $S(\mathrm{O}(2) \times \mathrm{O}(n-2))$ & $\mathrm{SO}(3)$ \\
$K_{2}$ & $S(\mathrm{O}(1) \times \mathrm{O}(n-1))$ & $S\left(\mathrm{O}(2)^{2}\right)$ \\
$K_{3}$ & $S(\mathrm{O}(3) \times \mathrm{O}(n-3))$ & $\mathrm{SO}(3)$ \\
\hline & & \\
\hline Group & $\mathrm{U}(n) \times \mathrm{SU}(n)$ & $\mathrm{Sp}(1) \times \mathrm{Sp}(n)$ \\
\hline Representation & $\rho_{3} \otimes \mu_{3} \otimes \mu_{n}$ & $v_{1} \otimes v_{n}$ \\
Angles & $\alpha_{1}=\pi / 2, \alpha_{2}=\pi / 4, \alpha_{3}=\pi / 3$ & $\alpha_{1}=\pi / 2, \alpha_{2}=\pi / 4, \alpha_{3}=\pi / 3$ \\
$H$ & $S\left(\mathrm{U}(1)^{3} \times \mathrm{U}(n-3)\right)$ & $\mathrm{Sp}(1)^{3} \times \mathrm{Sp}(n-3)$ \\
$L_{1}$ & $S(\mathrm{U}(1) \times \mathrm{U}(2) \times \mathrm{U}(n-3))$ & $\mathrm{Sp}(1) \times \mathrm{Sp}(2) \times \mathrm{Sp}(n-3)$ \\
$L_{2}$ & $S(\mathrm{U}(2) \times \mathrm{U}(1) \times \mathrm{U}(n-3))$ & $\mathrm{Sp}(2) \times \mathrm{Sp}(1) \times \mathrm{Sp}(n-3)$ \\
$L_{3}$ & $S\left(\mathrm{U}(1)^{2} \times \mathrm{U}(n-2)\right)$ & $\mathrm{Sp}(1)^{2} \times \mathrm{Sp}(n-2)$ \\
$K_{1}$ & $S(\mathrm{U}(2) \times \mathrm{U}(n-2))$ & $\mathrm{Sp}(2) \times \mathrm{Sp}(n-2)$ \\
$K_{2}$ & $S(\mathrm{U}(1) \times \mathrm{U}(n-1))$ & $\mathrm{Sp}(1) \times \mathrm{Sp}(n-1)$ \\
$K_{3}$ & $S(\mathrm{U}(3) \times \mathrm{U}(n-3))$ & $\mathrm{Sp}(3) \times \operatorname{Sp}(n-3)$ \\
\hline
\end{tabular}

6. Conclusions. Much has been written about isometric actions on spheres, and in particular, cohomogeneity-two actions have been studied by a great number of people. Here, we have tried to give accessible, explicit descriptions of how these orbit spaces and their underlying isotropy groups are computed.

A careful examination of all the actions leads to surprisingly few orbit spaces under these actions. The orbit spaces are homeomorphic either to a sphere or to a disk. The 
disks may have zero, one, two, or three vertices. The angles at these vertices may be $\pi / 2, \pi / 3, \pi / 4$, or $\pi / 6$. In the case of irreducible actions, the orbit spaces are limited to the spherical triangles $\Delta_{2,3,4}, \Delta_{2,3,3}$, and $\Delta_{2,2,3}$. These are all subsets of the two-sphere of radius 1 or $1 / 2$.

Some questions that remain in this area include:

(1) what are the orbit spaces associated with actions of cohomogeneity two on manifolds of strictly positive sectional curvature? It is well known [1] that these orbit spaces will be two-spheres or disks and we know geometrically that any such disk can have at most three vertices, so the question remains as to which angle configurations occur; and similarly,

(2) what are the orbit spaces associated with cohomogeneity-three actions on spheres (and on manifolds of strictly positive curvature)? In particular, the actions themselves are yet to be classified.

ACKNOwLEDGment. This work was supported in part by Consejo Nacional de Ciencia y Tecnologia, CONACYT project number 28491-E.

\section{REFERENCES}

[1] G. E. Bredon, Introduction to Compact Transformation Groups, Pure and Applied Mathematics, vol. 46, Academic Press, New York, 1972.

[2] R. Gilmore, Lie Groups, Lie Algebras, and Some of Their Applications, John Wiley \& Sons, New York, 1974.

[3] K. Grove and S. Halperin, Dupin hypersurfaces, group actions and the double mapping cylinder, J. Differential Geom. 26 (1987), no. 3, 429-459.

[4] W.-Y. Hsiang and H. B. Lawson Jr., Minimal submanifolds of low cohomogeneity, J. Differential Geometry 5 (1971), 1-38.

[5] W. Klingenberg, A Course in Differential Geometry, Graduate Texts in Mathematics, vol. 51, Springer-Verlag, New York, 1983.

[6] D. Montgomery, H. Samelson, and C. T. Yang, Exceptional orbits of highest dimension, Ann. of Math. (2) 64 (1956), 131-141.

[7] I. Satake, Linear Algebra, Pure and Applied Mathematics, vol. 29, Marcel Dekker, New York, 1975.

[8] E. Straume, On the invariant theory and geometry of compact linear groups of cohomogeneity $\leq$ 3, Differential Geom. Appl. 4 (1994), no. 1, 1-23.

[9]___ Compact connected Lie transformation groups on spheres with low cohomogeneity. I, Mem. Amer. Math. Soc. 119 (1996), no. 569, vi+93.

[10]___ Compact connected Lie transformation groups on spheres with low cohomogeneity. II, Mem. Amer. Math. Soc. 125 (1997), no. 595, viii+76.

[11] F. Uchida, An orthogonal transformation group of ( $8 k-1)$-sphere, J. Differential Geom. 15 (1980), no. 4, 569-574.

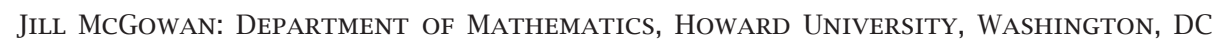
20059, USA

E-mail address: jmcgowan@howard. edu

Catherine Searle: Instituto de Mathemáticas de la UNAM, Unidad Cuernavaca, Avenida Universidad S/N, Colonia lo Mas de Chamilpa, Cuernavaca, Morelos 62210, MEXICO

E-mail address: csearle@matcver. unam . mx 


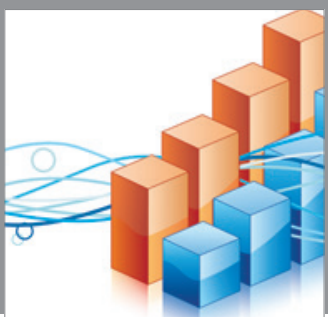

Advances in

Operations Research

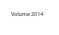

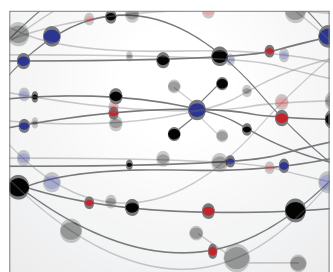

\section{The Scientific} World Journal
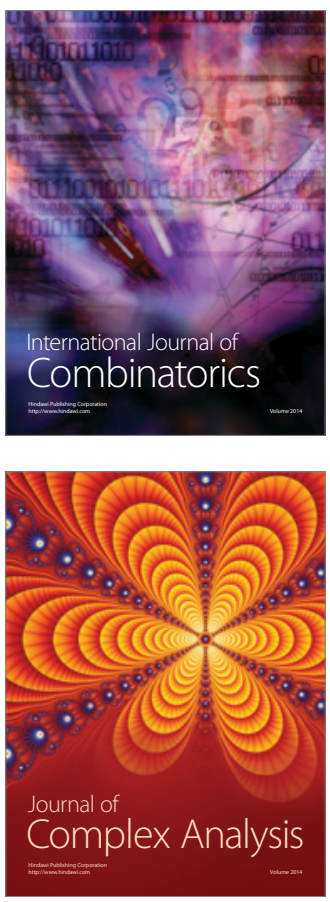

International Journal of

Mathematics and

Mathematical

Sciences
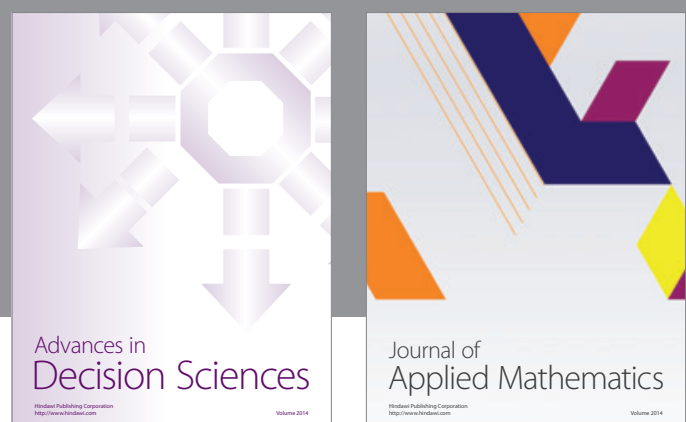

Journal of

Applied Mathematics
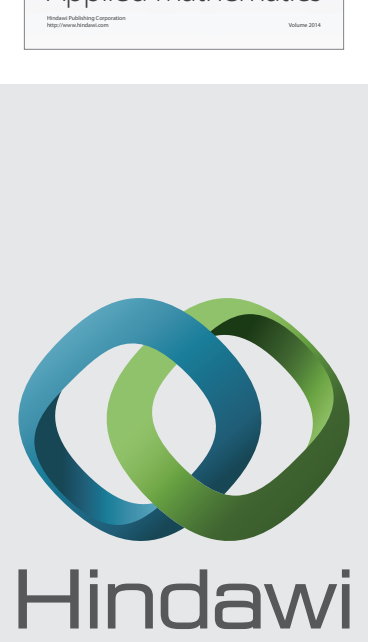

Submit your manuscripts at http://www.hindawi.com
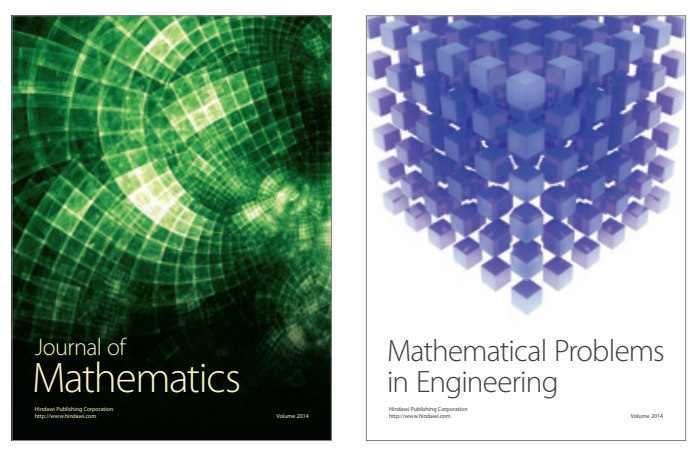

Mathematical Problems in Engineering
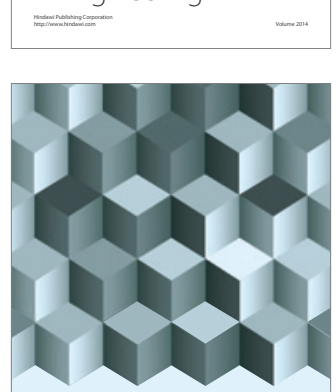

Journal of

Function Spaces
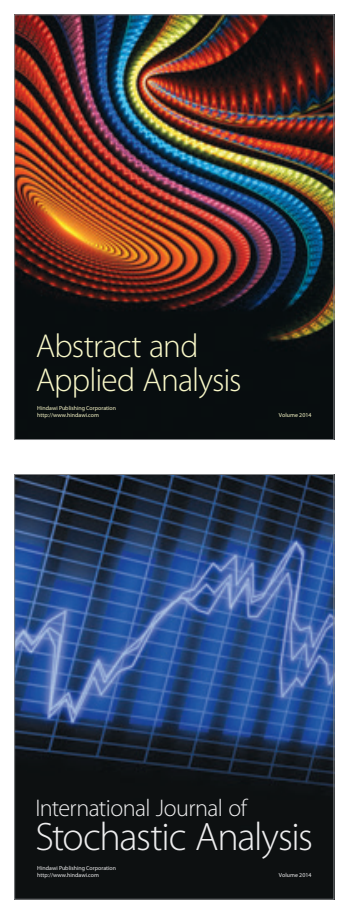

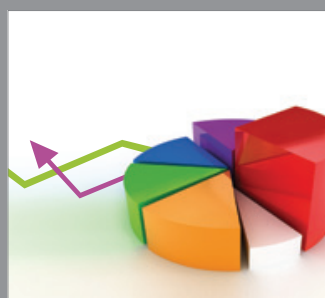

ournal of

Probability and Statistics

Promensencen
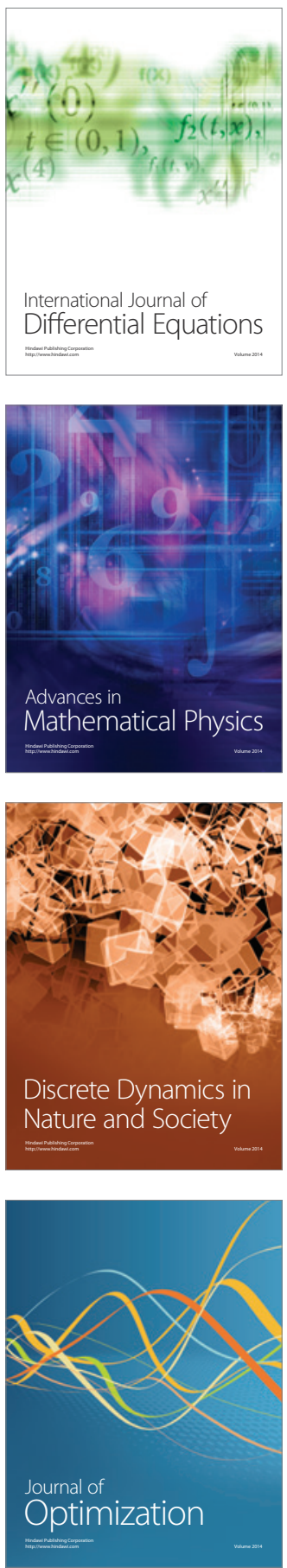